IAU Colloquium 164: Radio Emission from Galactic and Extragalactic Compact Sources

ASP Conference Series, Vol. 144, 1998

J. A. Zensus, G. B. Taylor, \& J. M. Wrobel (eds.)

\title{
An Update on Radio Supernovae
}

Schuyler D. Van Dyk ${ }^{1}$

Dept. Phys 8 Astr., UCLA, Los Angeles, CA 90095

Richard A. Sramek

National Radio Astronomy Observatory, Socorro, NM 87801, U.S.A.

Kurt W. Weiler \& Marcos J. Montes ${ }^{2}$

Code 7214, Naval Research Lab, Washington, DC 20375-5320, U.S.A.

Nino Panagia ${ }^{3}$

STSCI, Baltimore, MD 21218, U.S.A.

Abstract. The radio emission from supernovae ( $\mathrm{SNe}$ ) is nonthermal synchrotron radiation of high brightness temperature, with a "turn-on" delay at longer wavelengths, power-law decline after maximum with index $\beta$, and spectral index $\alpha$ asymptotically decreasing with time to a final, optically thin value. Radio supernovae (RSNe) are best described by the Chevalier (1982) "mini-shell" model, with modifications by Weiler et al. (1990). RSNe observations provide a valuable probe of the $\mathrm{SN}$ circumstellar environment and constraints on progenitor masses. We present a progress report on a number of recent $\mathrm{RSNe}$, as well as on new behavior from RSNe $1979 \mathrm{C}$ and $1980 \mathrm{~K}$, and on RSNe as potential distance indicators. In particular, we present updated radio light curves for SN $1993 \mathrm{~J}$ in M81.

\section{Radio Emission from Supernovae}

We have detected and are monitoring with the Very Large Array (VLA) radio emission from several recent supernovae, including SNe 1993J, 1994I (Rupen et al., in preparation), and 1996cb (Van Dyk et al., in preparation), bringing the total of radio supernovae ( $\mathrm{RSNe}$ ) studied by our group to 19 . In addition, we also have detected SN 1986E (Montes et al. 1997), and have discovered profound changes in the radio behavior of SNe $1979 \mathrm{C}$ (Montes et al., in preparation) and $1980 \mathrm{~K}$ (Montes et al., in preparation).

The radio behavior is best described by the Chevalier (1982) "mini-shell" model, as parameterized by Weiler et al. (1986, 1990). The observed synchrotron emission is generated by the $\mathrm{SN}$ shock interacting with a relatively high-density circumstellar medium (CSM), which has been fully ionized and heated by the initial UV/X-ray flash. This $\operatorname{CSM}\left(\rho_{\mathrm{CSM}} \propto r^{-2}\right)$ was presumably established by mass-loss episodes in the late stages of the progenitor's presupernova evolution.

Type Ib/c SNe turn on rapidly, reach a nearly common peak $6 \mathrm{~cm}$ luminosity, and decline rapidly thereafter; Type II SNe take much longer to turn on and to decline in the radio, and are heterogeneous in peak $6 \mathrm{~cm}$ luminosity. Type IIb RSNe may be intermediate cases, with properties between those of Type $\mathrm{Ib} / \mathrm{c}$ and Type II RSNe. Type Ia SNe have not been detected to the sensitivity limit of the VLA.

\footnotetext{
${ }^{1}$ Visiting scientist.

${ }^{2} \mathrm{Naval}$ Research Lab/National Research Council Cooperative Research Associate

${ }^{3}$ Also affiliated with ESA
} 


\section{2. $\mathrm{SN} 1993 \mathrm{~J}$ in $\mathrm{M} 81$}

Radio data for the Type IIb SN 1993J in M81, shown in Fig. 1, exhibit regular evolution through 1997 January, similar to the behavior found by Van Dyk et al. (1994).

The standard Chevalier model, as applied to all other RSNe, provides a very poor fit to the data for SN 1993J. The fit we do find implies that the absorbing medium is strictly external, with both uniform and clumpy, "mixed" components, and that $\rho_{\mathrm{CSM}} \propto r^{-3 / 2}$, i.e., shallower than our standard assumption. Such a profile can be obtained if the mass-loss rate, $\dot{M}$ (for a constant wind velocity, $w$ ), was decreasing just prior to explosion. The standard Chevalier model clearly needs modification to include more physics and new phenomena.

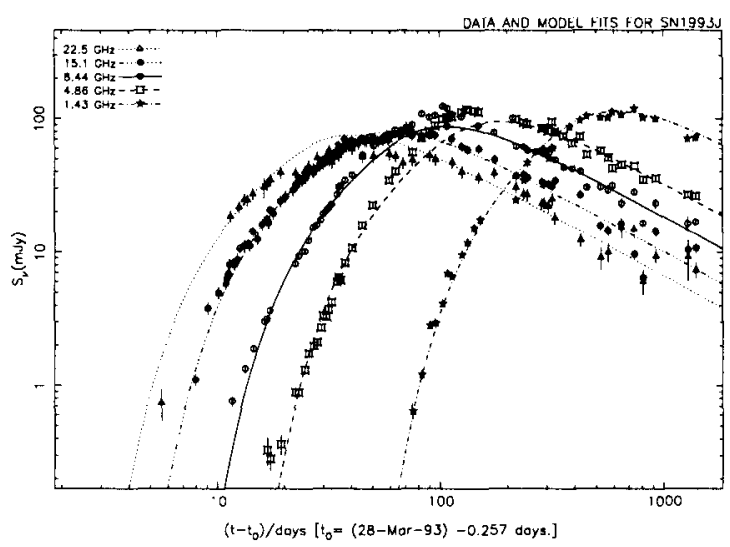

Figure 1. The radio light curves for SN 1993J. VLA continuum data are $1.3 \mathrm{~cm}$ (triangles), $2 \mathrm{~cm}$ (circles with crosses), $3.6 \mathrm{~cm}$ (circles), $6 \mathrm{~cm}$ (squares), and $20 \mathrm{~cm}$ (stars); the $2 \mathrm{~cm}$ points include data from the Ryle Telescope (c.f., Pooley \& Green 1993). The data since 1993 November $28 \approx$ day 250 are not in Van Dyk et al. (1994).

Acknowledgments. SVD was supported by a grant from NASA administered by the AAS. KWW and MJM are supported by 6.1 funding from the Office of Naval Research. The National Radio Astronomy Observatory is a facility of the NSF, operated under a cooperative agreement. by Associated Universities, Inc.

\section{References}

Chevalier, R. A. 1982. ApJ, 259, 302-310.

Montes, M. J., et al. 1997. $A p J, 482$, L61-64.

Pooley, G. G., \& Green, D. A. 1993. MNRAS, 264, L17-20.

Van Dyk, S. D., et al. 1994. ApJ, 432, L115-118.

Weiler, K. W., Panagia, N., \& Sramek, R. A. 1990. ApJ, 364, 611-625.

Weiler, K. W., et al. 1986. ApJ, 301, 790-812. 